\title{
Ambulatory postural blood pressure changes and history allow a better selection of patients that should undergo a head-up tilt test
}

\author{
Enrique Asensio ${ }^{1}$, Melisa Lecuna ${ }^{2}$, Eliodoro Castro ${ }^{3}$, Jose Benito Alvarez ${ }^{4}$, \\ Susano Lara ${ }^{5}$, Humberto Castro ${ }^{5}$, Leonor Mendoza ${ }^{3}$, Nidia Cordero $^{3}$ \\ ${ }^{1}$ Cardiology, Electrophysiology Head, Medicine Department, Hospital Medica TEC 100, Mexico \\ ${ }^{2}$ Universidad del Valle de Mexico, Mexico \\ ${ }^{3}$ Instituto de Corazon de Queretaro, Mexico \\ ${ }^{4}$ Hospital Espanol de Mexico, Mexico \\ ${ }^{5}$ Instituto Mexicano del Seguro Social, T1 Leon Guanajuato, Mexico
}

\begin{abstract}
Background: Syncope is a common symptom and in most cases it is of a neurally mediated origin. Such patients have to be studied with a careful history and a physical exploration that should include simple maneuvers such as blood pressure (BP) recordings in decubitus and standing position. These tools can suggest diagnosis in a good percentage of patients without the need for expensive or invasive testing.

Methods: We carried out a prospective observational study measuring BP and heart rate (HR) with the patients in decubitus and just as they stood up. The patients were sent for a tilt table test in different specialized centers. The BP changes were compared to the results of the tilt test.

Results: We included 215 patients, $36.1 \pm 18.8$ years old, 118 (54.9\%) feminine, of which 143 (66.5\%) had a positive tilt test. Patients with a positive test showed a rise in systolic BP $(S B P)(121.7 \pm 19.1$ vs. $124.2 \pm 20, p<0.005)$ and in diastolic $B P(D B P)(75 \pm 11$ vs. $78 \pm 11.3, p<0.005)$ when compared to people with a negative one. On the other hand, percentage BP changes were significantly different (SBP 2.24\% vs. $0.48 \%, p=0.02 ; D B P 4.1 \%$ vs. $1.2 \%, p=0.009)$. Patients with a positive test had also a lower HR on standing up (72.1 \pm \pm 11.1 vs. $78.3 \pm 17.2, p=0.01$ ).
\end{abstract}

Conclusions: Patients with neurally mediated syncope showed an elevation of SBP and DBP when standing up actively, unlike subjects with a negative tilt test. (Cardiol J 2015; 22, 2: 165-171)

Key words: neurocardiogenic syncope, blood pressure, active standing, head-up tilt table test

\section{Introduction}

Syncope is a common problem. Its prevalence is high and it is a common symptom among young and elderly people. The etiologies of syncope are diverse through life, but one of the most common is neurally mediated syncope, also known as neurocardiogenic [1]. Syncope recurrence rate is

Address for correspondence: Dr Enrique Asensio, Cardiology, Electrophysiology Head, Medicine Department, Hospital Medica TEC 100, Mexico, e-mail: easensiol@gmail.com 
variable according to the origin of the symptom, but it is known that from $40 \%$ to $85 \%$ of people who have had an episode of syncope and look for medical attention will not have a recurrent episode [2].

This is important because syncope needs to be carefully evaluated when present, and it should be done according to the general context of the patient. When syncope appears in adults with structural heart disease, the symptom usually implies a bad prognosis. In young people with normal hearts, syncope is usually a benign condition, although the clinical evaluation must include an electrocardiogram (ECG) not only to assess ventricular hypertrophy but also channelopathies or other conditions.

Current syncope guidelines establish the indications for the use of different diagnostic tests $[1,3]$. A thorough history, careful physical exam and an ECG are the initial diagnostic tools and they provide a good diagnostic yield as well as a possibility to define the best study plan for the patient. Yet, in daily practice it seems that many simple measurements that could enhance the initial evaluation are omitted, especially when the patient is not evaluated in specialized setting or a syncope unit [4-7]. Many times costly tests or even invasive ones are ordered without a complete evaluation of the patient with simple measures.

On the other hand, several studies have tried to make an early prediction of the head-up tilt table test (HUTT) result, nevertheless, the results remain contradictory and there is no single measurement or combination that are able to predict the HUTT's result with precision [8-13].

At the moment, the HUTT can be considered the best available means for neurally mediated syncope diagnosis when the history and physical exam have not been enough. Nevertheless, its sensitivity, specificity and predictive values continue to be debated since there is no gold standard to compare it with. This is why it is important to carefully select the subjects that will undergo a HUTT, aside from having more precise diagnostics and avoiding unnecessary risks and costs.

Blood pressure (BP) measurement in decubitus and standing position, as well as carotid sinus massage are simple maneuvers that can be performed under ECG monitoring at the office. These can support the history and physical exam findings, thus allowing better selection of the subjects that should undergo a HUTT or other tests such as echocardiography, prolonged rhythm monitoring or invasive tests to search for arrhythmias.

\section{Methods}

A prospective, cross-sectional study was performed to evaluate if there was any relationship between the BP postural changes recorded in the office and the result of a HUTT.

Patients of both genders between 10 and 75 years old referred to two centers for a HUTT were included. All the patients were in a syncope study protocol, mainly referred by general practitioners, internal medicine specialists and cardiologists.

Before the HUTT, the patients came to the office in comfortable clothes and were given a $5 \mathrm{~min}$ rest period in decubitus. Then the basal BP recording was obtained with a standardized technique and an aneroid sphygmomanometer. The heart rate (HR) was determined by radial pulse frequency for $15 \mathrm{~s}$.

Once these two measurements were complete, the patient stood up by his own means and immediately we obtained a new BP measurement in the same arm with the same device and a new $\mathrm{HR}$ after completing the $\mathrm{BP}$ recording.

After these measurements were obtained, the patient was taken to the HUTT room where he was prepared for the test by a nurse. While the patient was being prepared, a history about syncope episodes was obtained. The investigators knew the HUTT result at the end of the procedure. The diagnosis included in the report was the one obtained by the physician in charge of the test, but the investigators were excluded from the HUTT as well as the physician in charge was unaware of the $\mathrm{BP}$ and $\mathrm{HR}$ changes prior to the test.

The HUTT protocol was a responsibility of the physician in charge of the test, albeit a standardized one was used as described in the 2009 European guidelines [1]. Both hospitals agreed to use the same standardized protocol that included two tilt phases. The patient was placed on a tilt table, monitored and an IV access was obtained. After a $10 \mathrm{~min}$ rest, the patient was passively placed in a $70^{\circ}$ tilt angle for the first $30 \mathrm{~min}$ passive tilt. If no diagnosis was reached, the patient was again put in a decubitus position and was given 2.5 to $5 \mathrm{mg}$ dinitrate isosorbide spray sublingually (the dosage was calculated according to the patients' weight). The subject was again positioned in a $70^{\circ}$ degree tilt position for another $20 \mathrm{~min}$ or less if they presented symptoms. The diagnostic criteria were the ones mentioned in the European guidelines and in the Mexican Guidelines [1, 3].

The whole procedure was explained to the patients before the basal measurements, when they were invited to participate in the study. If the pa- 
Table 1. General data of the studied population according to the result of the head-up tilt test (HUTT).

\begin{tabular}{|c|c|c|c|}
\hline & $\begin{array}{c}\text { Positive HUTT } \\
(n=143,66.5 \%)\end{array}$ & $\begin{array}{l}\text { Negative HUTT } \\
(\mathrm{n}=72,33.5 \%)\end{array}$ & $\mathbf{P}$ \\
\hline Female gender & $80(55.9 \%)$ & $38(52.8 \%)$ & 0.45 \\
\hline Age [years] & $35.2 \pm 19.2$ & $37.08 \pm 18.5$ & 0.7 \\
\hline \multicolumn{4}{|l|}{ Syncope associated with: } \\
\hline Closed space & $14(9.8 \%)$ & $9(12.5 \%)$ & 0.23 \\
\hline Emotional stress & $15(10.5 \%)$ & $13(18.1 \%)$ & 0.002 \\
\hline Heat & $13(9.1 \%)$ & $6(8.3 \%)$ & 0.71 \\
\hline Exercise & $8(5.6 \%)$ & $17(23.6 \%)$ & $<0.0005$ \\
\hline Evolution time [years] & $9.7 \pm 11.4$ & $8.7 \pm 7.8$ & 0.007 \\
\hline Number of previous syncope episodes & $3.2 \pm 1.5$ & $3.3 \pm 1.2$ & 0.09 \\
\hline Nitrate — potentiated HUTT & $70(49 \%)$ & $64(88.9 \%)$ & 0.0005 \\
\hline \multicolumn{4}{|l|}{ Symptoms referred in the previous history: } \\
\hline Nausea & $76(53.12 \%)$ & $21(29.2 \%)$ & $<0.0005$ \\
\hline Dizziness & $105(73.4 \%)$ & $41(56.9 \%)$ & $<0.0005$ \\
\hline Diaphoresis & $88(57.3 \%)$ & $30(41.7 \%)$ & 0.77 \\
\hline Visual blurring & $67(46.9 \%)$ & $35(48.6 \%)$ & 0.68 \\
\hline Symptom reproduction during HUTT & $120(83.9 \%)$ & $24(33.3 \%)$ & $<0.0005$ \\
\hline
\end{tabular}

tient accepted, he signed an informed consent form and the BP and HR measurements were obtained.

Patients who declined the invitation were not included. All the patients that did not have a diagnostic or complete HUTT, those with incomplete HUTT reports, those who could not assume a standing position on their own and the patients that had already been prepared for the HUTT (with an IV line and ECG monitoring) before obtaining baseline measurements were excluded from the study.

The protocol was evaluated and accepted by the Research and Ethics Committees of the Universidad del Valle de México campus Querétaro.

The results were analyzed with the SPSS 19 software package (IBM SPSS 2010, IBM Corporation, Armonk, New York 10504-1722, United States). Continuous variables are expressed as mean \pm standard deviation and the categorical ones as absolute numbers and percentages. The Student's t test and $\chi^{2}$ were used for comparison between groups. A logistic regression analysis was performed to evaluate possible associations between the BP changes and the HUTT's result.

\section{Results}

During a period of 14 months, 215 patients referred for HUTT were recruited in two different centers. One-hundred and eighteen (54.9\%) patients were female, the group's mean age was $36.1 \pm 18.8$ years.

None of the patients had a significant cardiovascular disease history. One-hundred and eighty-six (86.5\%) patients were studied because of unknown origin syncope, 29 were sent to a HUTT because of pre-syncope. The baseline ECG's were normal in the 215 subjects, but no dynamic tests were performed to look for ischemic heart disease, channelopathies or other diseases in the preparation for HUTT. It is noteworthy that the syncope study protocol was in charge of the referring physician, the group performing the HUTT and BP measures was not aware of the complete patient's history. We did not have access to complimentary tests such as echocardiograms, neurological evaluations or long-term ECG monitoring.

One-hundred and forty-three (66.5\%) patients had a positive HUTT. Table 1 shows the main demographics as well as the most important history data. Most subjects had recurrent syncope for more than 6 years.

The symptoms associated with syncope showed significant differences regarding the most commonly associated to it (nausea, diaphoresis, dizziness), but not in other data such as blurred vision or palpitations, for example.

The HUTT's results according to the VASIS classification are shown in Table 2 . The test was positive in the minute $26.7 \pm 16.7$ and in $49 \%$ of the cases it was necessary to use pharmacological stimulation with nitrate (isosorbide di-nitrate). In $83.9 \%$ of the patients with positive test, symptoms as dizziness, nausea, diaphoresis, palpitations or blurred vision were reproduced. In the group of negative tests this reproduction only happened in $33.3 \%$ of the subjects that neither showed any hemodynamic changes. 
Table 2. Head-up tilt test results according to the VASIS classification.

\begin{tabular}{lc}
\hline Result & $\mathbf{N}(\%)$ \\
\hline VASIS I & $57(26.5 \%)$ \\
VASIS Ila & $3(1.4 \%)$ \\
VASIS IIb & $11(5.1 \%)$ \\
VASIS III & $72(50.3 \%)$ \\
\hline
\end{tabular}

The symptoms described by the patients during the HUTT showed significant differences regarding headache, that was more prevalent in the negative HUTT group (47.2\% vs. $31.5 \%$, $\mathrm{p}=0.001)$. The group with positive HUTT had a higher frequency of dizziness, nausea, blurred vision, palpitations and anxiety, among others, with a significant $\mathrm{p}$ value $<0.005$.

Table 3 shows the absolute and percentage changes in $\mathrm{BP}$ and $\mathrm{HR}$ and compares the group with positive HUTT against the one with negative HUTT as well as the decubitus (baseline) and standing (minute 0) BP and HR measurements.
The logistic regression analysis did not show a significant increase in the risk of having a positive HUTT with the absolute changes in BP, but when the symptoms were added to the increase in systolic BP (SBP) and diastolic BP (DBP), the risk became higher and the differences reached significant $\mathrm{p}$ value (RR 5.5, 95\% CI 1.6-18.7, $\mathrm{p}=0.005)$. Table 4 shows the regression model.

\section{Discussion}

A systematic approach to the diagnosis of the patient with syncope, that is, a protocol for its study, is associated with a reduction in study costs and in an adequate use of the diagnostic tools. The clinical evaluation establishes the possible diagnosis in a variable percentage of syncope patients, and facilitates the decision making regarding what complimentary tests are more suitable for the patient. This initial clinical evaluation must be complete and should include the measurement of the BP in different positions at the office [1, 14-17].

The actual series of patients referred for a HUTT by a heterogeneous group of physicians

Table 3. Blood pressure changes induced by posture shift according to the result of the head-up tilt test (HUTT).

\begin{tabular}{lccc}
\hline & $\begin{array}{c}\text { Positive HUTT } \\
\text { (n = 143) }\end{array}$ & $\begin{array}{c}\text { Negative HUTT } \\
\text { (n = 72) }\end{array}$ \\
\hline Decubitus HR & $72.1 \pm 11$ & $79.3 \pm 20.6$ & 0.001 \\
Stand-up HR & $72 \pm 11.1$ & $78.3 \pm 17.2$ & 0.01 \\
P value comparing decubitus HR vs. stand-up HR & 0.8 & 0.44 & 0.3 \\
Decubitus SBP & $121.7 \pm 19.1$ & $119.2 \pm 11$ & 0.1 \\
Stand-up SBP & $124.2 \pm 20$ & $120 \pm 14$ & 0.25 \\
P value comparing decubitus SBP vs. stand-up SBP & $<0.005$ & $74.8 \pm 10.5$ & 0.8 \\
Decubitus DBP & $75 \pm 11$ & $75.5 \pm 10.1$ & 0.1 \\
Stand-up DBP & $78 \pm 11.3$ & 0.15 & 0.7 \\
P value comparing decubitus DBP vs. stand-up DBP & $<0.005$ & $0.2 \%$ & $0.48 \%$ \\
Percentage HR change decubitus vs. stand-up & $0.3 \%$ & $1.2 \%$ & 0.02 \\
Percentage SBP change decubitus vs. stand-up & $2.24 \%$ & $4.1 \%$ & 0.009 \\
Percentage DBP change decubitus vs. stand-up & & 0.1 & \\
\hline
\end{tabular}

*P value comparing positive vs. negative test; DBP — diastolic blood pressure; HR — heart rate; SBP — systolic blood pressure

Table 4. Logistic regression model.

\begin{tabular}{lccccc}
\hline & Beta & Error & Exp $(\beta)$ & $95 \%$ Cl & P \\
\hline Symptoms + SBP increase & 0.783 & 0.44 & 2.18 & $0.9-5.2$ & 0.07 \\
Symptoms + DBP increase & 4.22 & 0.42 & 1.52 & $0.6-3.4$ & 0.3 \\
Symptoms + SBP and DBP increase & 1.71 & 0.62 & 5.56 & $1.6-18.7$ & 0.005 \\
\hline
\end{tabular}

$\mathrm{Cl}$ - confidence interval; DBP — diastolic blood pressure; SBP — systolic blood pressure 
show adults with demographic characteristics similar to those described by other authors. The gender distribution and the positive HUTT rate are also within the known ranges.

It is noteworthy that, regarding the circumstances around the syncope episode, the significant differences happened when syncope was associated to emotional stress and exercise-related syncope.

Emotional stress can be related with anxiety crisis or psychogenic syncope, two conditions that usually have a good long-term prognosis. A careful history and consecutive BP measurements after hyperventilation, the Valsalva maneuver and postural change can avoid performing a test that will not provide further information on these subjects.

On the other hand, the subjects that have an exercise-related syncope represent a high risk subset of patients that must be studied to detect structural heart disease and lethal arrhythmias $[1,4,18-20]$. Thus, sudden cardiac death risk stratification is mandatory and the HUTT is not a test designed to identify the mentioned high risk conditions. The specific question to the patients was if they had a syncope episode while exercising, so the BP measurements in different postures would probably have not brought any new relevant information. In this context, the patient can get more specific tests before having a HUTT that in an initial approach is not a valuable test and can delay the correct diagnosis. Of course, there are subjects in this setting that can benefit from a HUTT, but once a high risk condition has been ruled out.

Symptom reproduction during HUTT is now a diagnostic criterion as important as the hemodynamic changes [1]. Most patients with a positive HUTT showed a reproduction of their symptoms, while the patients with a negative test did not. The latter had more headaches, possibly because of the higher nitrate dose.

These data reinforce the usefulness of the medical history and a directed interrogation. The combination of symptoms such as nausea, dizziness and diaphoresis had already been studied and it was found to have a good correlation with the HUTT result [10]. In this group, such a behavior is reproduced and that is why it was included in the regression analysis.

The early changes in BP and HR have a controversial value as early predictors of the HUTT's result. Today, there is a consensus regarding their poor predictive value for the test result (positive or negative HUTT) as well as for the sort of neurally mediated syncope reproduced $[8,9,11-13,21]$.

Nevertheless, in a previous study it was found that the three abovementioned symptoms combined with a reduction in SBP and an increase of less than $1 \%$ for the DBP at the moment of finishing the tilt of the patient was associated with a higher rate of positive HUTT [11]. In the present study, a different phenomenon was found: The subjects with a positive test had a slight increase in SBP and a more noticeable one in DBP. When these $\mathrm{BP}$ changes were included in the regression model alongside with the described symptoms, the risk of having a positive HUTT was significantly increased.

This hemodynamic behavior is different from the one observed before, and possibly it has to do with the way the patient assumed the standing position. In the previous study, the patient was already in the tilting table and was passively taken to a $70^{\circ}$ angle. In the present study, the patient had to assume the standing position by his own means.

The physiologic difference of assuming an erected position in a passive or active manner has been addressed by several authors and in some cases the differences are not clear.

When the person stands up actively, the contraction of the different muscular groups in the legs, abdomen and back induces an initial increase in $\mathrm{BP}$ that compensates for the volume redistribution: The increase of the venous return towards the right atrium induced by the contraction of big muscular groups increases the left ventricle's preload in healthy subjects, and thus there is a momentarily higher SBP. The DBP raise can be explained by the muscular contraction that increases peripheral vascular resistances. Subjects with syncope might probably have a sympathetic hyperactivity that makes those hemodynamic changes more notorious. These changes could probably be detected with continuous recording instruments ("finometry") or through HR variability analysis, but such instruments are not usually found in a common office [22-25]. The fact that the most noticeable changes happen in DBP suggest that the peripheral autonomic nervous system is more active regulating vascular tone than $\mathrm{HR}$, at least in the first moments of the voluntary position shifting.

The small variations of HR are striking but there are several possible explanations. The first aspect to consider is the methodology to obtain the HR, since BP and HR measurements were not performed simultaneously on standing up and the BP measurement was privileged at the "minute 0". The measurements performed in other studies showed that the HR differences become significant after several minutes of tilt or standing up. One study found that even in the postural orthostatic 
tachycardia syndrome, there were not significant differences in HR when the patient assumed the upright position actively or passively [26, 27]. Previous studies have explored the discriminatory abilities of the HR changes to predict the result of the HUTT, as has been said before, but there are non-conclusive results $[8,9]$.

Another possible explanation is that the lack of HR changes is derived from a primary set of vascular events. That is, the high sympathetic activity possibly alters HR variability, but a basal pulse recording is unable to discriminate such a condition. Thus, the postural change induces the described phenomena regarding venous return, without a significant change in $\mathrm{HR}$, although it could possibly influence the left ventricle's contractility because of the increase in end-diastolic volume. The difference in HR between the positive and negative HUTT is harder to explain based on the hypothesis of an enhanced sympathetic tone among syncope patients. The complete assessment might go again through the evaluation of HR variability or QT dispersion.

Finally, the hypothesis that the autonomic nervous system might be more active in the peripheral vascular system or that a continuous stimulation of heart ganglia might blunt the HR response, partially explains the abovementioned differences. Pachon et el. [28, 29] have described how the destruction of the para-sympathetic neurons in the atrial myocardium can modulate the HR response to the sympathetic - para-sympathetic stimulation avoiding severe bradycardia related to neurally mediated syncope. The results of cardioneuroablation have been encouraging regarding the control of slow HRs in such patients, although they have not showed any benefit regarding BP control. This can partially support the hypothesis of a blunted HR response and of a differential effect of the autonomic nervous system according to the effector organ [28, 29]. This opens new research possibilities to evaluate a differential behavior of the autonomic nervous system.

On the other hand, the present study stretches the need for a complete evaluation of the patient with syncope in a syncope unit. It is relevant not only from a research point of view, but mainly because of the need for a complete evaluation of the patient. As we have discussed earlier, some patients had had a high risk syncope episode, and even if the mean evolution time and mean number of episodes suggest a benign origin for the symptom, the fact is that some patients would have needed a different approach [30].

\section{Conclusions}

These results show that there are different behaviors among patients with neurally mediated syncope and persons with syncope from a different etiology.

Apparently, the increases in BP are the consequence of a hyper-reactive sympathetic activity that is on line with the current pathophysiological theories of neurally mediated syncope. The changes in the BP, and especially the percentage changes, that are statistically significant, could be a helpful tool to define what patients can be more suitable to undergo a HUTT. The people with a neurocardiogenic syncope history, with a normal ECG that show the described changes in BP probably will not require a HUTT unless it is valuable for them to demonstrate the dysautonomic origin of the symptom, as mentioned in the European guidelines [1].

Patients without these changes and with an unknown origin syncope should continue to be studied with a HUTT when considered appropriate, albeit it must be said that young people with exercise related syncope must be initially studied by other means to define the sudden cardiac death risk before searching a neurally mediated syncope. The integrative view of a syncope unit can help to lessen the risks of a non-protocoled approach.

\section{Conflict of interest: None declared}

\section{References}

1. Moya A, Sutton R, Ammirati F et al. Guidelines for the diagnosis and management of syncope (version 2009). Eur Heart J, 2009; 30: 2631-2671.

2. Kenny RA, Ingram A, Bayliss J, Sutton R. Head-up tilt a useful test for investigating unexplained syncope. Lancet, 1996; 1: 1352-1354.

3. Asensio E, González Hermosillo JA, Rodríguez LL. El manejo del síncope: Diagnóstico y tratamiento. In: Miembros de la Junta Directiva SOMEEC 2007-2009, Guías de práctica médica de la Sociedad Mexicana de Electrofisiología y Estimulación Cardiaca, 2009: pp. 70-92.

4. Linzer M, YangEH, Estes NA3rd, Wang P, Vorperian VR, KapoorWN. Diagnosing syncope. Part 1: Value of history, physical examination, and electrocardiography. Clinical Efficacy Assessment Project of the American College of Physicians. Ann Intern Med, 1997; 126: 989-996.

5. Farwell DJ, Sulke AN. Does the use of a syncope diagnostic protocol improve the investigation and management of syncope? Heart, 2004; 90: 52-58.

6. Brignole M, Malasana G, Sherwood RP, Daccarett M, Jetter TL, Hamdan MH. Evaluation of patients with "faint" in an American teaching hospital: A dire need for a standardized approach. Pacing Clin Electrophysiol, 2011; 34: 284-290. 
7. Ungar A, Morrione A, Rafanelli M et al. Evaluation of complex syncope: what are the indications for second-level investigations? G Ital Cardiol, 2009; 10: 6-17.

8. Alvarez B, Asensio E, Lozano E, Portos JM. Early heart rate variations during the Head-up tilt table testing as a predictor of the outcome the test. Pacing Clin Electrophysio. Ene, 2000; 23: 25-31.

9. García A, Lacunza J, Rojo J et al. Los cambios tempranos en la frecuencia cardiaca no predicen el resultado de la prueba de mesa basculante potenciada con nitroglicerina. Rev Esp Cardiol, 2005; 58: 499-503.

10. Asensio E, Oseguera J, Loría A et al. Clinical findings as predictors of positivity of head-up tilt table test in neurocardiogenic syncope. Arch Med Res, 2003; 34: 287-291.

11. Asensio E, Colín E, Castillo L et al. Comportamiento diferencial de la tensión arterial de pacientes con síncope neurocardiogénico en la fase inicial de la prueba de inclinación. Arch Inst Cardiol Mex, 2006; 76: 59-62.

12. Gielerak G, Guzik P, Makowski K, Kowal J, Cholewa M. Haemodynamic indices of the early phase of the tilt test: does measurement predict outcome? Kardiol Pol, 2005; 63: 244-251.

13. Turk U, Alioglu E, Kirilmaz B et al. Prediction of head-up tilt test result: Is it possible? Pacing Clin Electrophysiol, 2010; 33: 153-158.

14. Dupliakov DV, Golovina GA, Sysuenkova EV, Glukhova VL. [Assessment of vasovagal origin of syncope based on history features]. Kardiologiia, 2012; 52: 55-60.

15. Kapoor WN, Karpf M, Maher Y, Miller RA, Levey GS. Syncope of unknown origin. The need for a more cost-effective approach to its diagnosis evaluation. JAMA, 1982; 247: 2687-2691.

16. Krahn AD, Klein GJ, Yee R, Hoch JS, Skanes AC. Cost implications of testing strategy in patients with syncope: Randomized assessment of syncope trial. J Am Coll Cardiol, 2003; 42: 495-501.

17. Steinberg LA, Knilans TK. Syncope in children: Diagnostic tests have a high cost and low yield. J Pediatr, 2005; 146: 355-358.

18. Gallagher J, Huang DT. Wilde AA, Rosero SZ. Syncope and exercise-related ventricular tachycardia. Ann Noninvasive Electrocardiol, 2012; 17: 146-150.

19. Adeoye AM, AdekunleAN, Adebiyi AA, MullassariA, VijayakumarS, Nwafor CE. A 45-year old man with recurrent syncope: An unu- sual presentation of coronary artery disease. Pan Afr Med J, 2013; $19: 71$.

20. Roses Noguer F, Jarman JW, Clague JR, Till J. Outcomes of defibrillator therapy in catecholaminergic polimorphyc ventricular tachycardia. Heart Rhythm, 2014; 11: 58-66.

21. Mallat Z, Vicaut E, Sangaré A, Verschueren J, Fontaine G, Frank R. Prediction of head-up tilt test result by analysis of early heart rate variations. Circulation, 1997; 96: 581-584.

22. Wieling W, Krediet CT, van Dijk N, Linzer M, Tschakovsky ME. Initial orthostatic hypotension: Review of a forgotten condition. Clin Sci, 2007; 11: 157-165.

23. Malamud-Kessler C, Estañol-Vidal B, Infante-Vázquez Ó, Campos-Sánchez M, Chiquete E. [Baroreflex behavior in patients with vasovagal syncope during active standing]. Rev Neurol, 2013; 57: 199-206.

24. Chladekova L, Czippelova B, Turianikova Z et al. Multiscale time irreversibility of heart rate and blood pressure variability during orthostasis. Physiol Meas, 2012; 33: 1747-1756.

25. Holmegard HN, Benn M, Kaijer M, Haunsø S, Mehlsen J. Differences in autonomic balance in patients with cardioinhibitory and vasodepressor type of reflex syncope during head-up tilt test and active standing. Scand J Clin Lab Invest, 2012; 72: 265-273.

26. Davis SC, Westerhof BE, van den Bogaard B et al. Active standing reduces wave reflection in the presence of increased peripheral resistance in young and old healthy individuals. J Hypertens, 2011; 29: 682-689.

27. Kirbiš M, Grad A, Meglič B, Bajrović FF. Comparison of active standing test, head-up tilt test and 24-h ambulatory heart rate and blood pressure monitoring in diagnosing postural tachycardia. Funct Neurol, 2013; 28: 39-45.

28. Pachon J, Pachon E, Cunha M, Lobo T, Pachon J, Santillana T. Catheter ablation of severe neurally mediated reflex (neurocardiogenic or vasovagal) syncope: Cardioneuroablation long-term results. Europace, 2011; 13: 1231-1242.

29. Pachon JC, Pachon EI, Pachon JC et al. "Cardioneuroablation" new treatment for neurocardiogenic syncope, functional AV block and sinus dysfunction using catheter RF ablation. Europace, 2005; 7: 1-13.

30. Vigar S, Bradley D, Shen W. Syncope units: impact on patient care and health related costs. Cardiol Clin, 2013; 31: 39-49. 